\title{
Transcriptome of hypertension-induced left ventricular hypertrophy and its regression by antihypertensive therapies
}

\author{
Julio Gallego-Delgado ${ }^{1,4,5}$, Susan B Connolly ${ }^{2,5}$, Alberto Lázaro ${ }^{3,4}$, Denise Sadlier ${ }^{2}$, Niamh E Kieran ${ }^{2}$, \\ Declan D Sugrue ${ }^{2}$, Peter Doran ${ }^{2}$, Hugh R Brady ${ }^{2}$, Julio Osende ${ }^{1,5}$ and Jesus Egido ${ }^{4,5}$
}

Left ventricular hypertrophy (LVH), a common consequence of systemic hypertension associated with poor clinical outcome, is also a potentially reversible condition. Here, we probed the molecular pathways that underpin the development of LVH and their modulation by antihypertensive regimens that reversed LVH. Spontaneously hypertensive rats were studied at 12 (early LVH) and 48 weeks (late LVH), respectively, with normotensive Wistar-Kyoto rats as age-matched controls. Three treatment groups were maintained for 36 weeks on the following regimens: (1) quinapril, (2) doxazosin and quinapril combination, and (3) losartan. Gene expression profiling was performed with Affymetrix microarrays (GeneChip Rat-230A) and primary function-focused average linkage hierarchical cluster analysis. Of the 15696 gene sequences expressed on the Affymetrix GeneChip Rat-230A, there was significant alteration in the expression of $295(1.9 \%)$ of these transcripts in 'early' LVH and $143(0.9 \%)$ in 'late' LVH. The predominant changes in gene expression were seen in metabolism, cell growth/proliferation, signal transduction, development and muscle contraction/cytoskeleton functional groups. Although sharing many effects on gene expression, the three treatments showed different expression profiles. Despite significant regression of LVH with treatment, $31 \mathrm{LVH}$-associated transcripts were unchanged by any of the treatment groups. Our data suggest that LVH regression does not normalize the LVH transcriptome. Therefore, regression of hypertension-induced LVH is associated with a distinct gene expression profile, suggesting the effect of both treatment and a previously unknown specific myocardial physiology after regression of LVH. Hypertension Research (2009) 32, 347-357; doi:10.1038/hr.2009.27; published online 27 March 2009

Keywords: antihypertensive agents; cardiac hypertrophy; gene expression profiling

\section{INTRODUCTION}

Left ventricular hypertrophy (LVH) is considered an adaptive response of the heart to a variety of stresses, including pressure overload. This condition is associated with a significant mortality and also, with a higher risk for the development of coronary heart disease, myocardial infarction and stroke, ${ }^{1}$ is considered to be the single most powerful predictor of heart failure in Western societies. ${ }^{2}$ The molecular pathways that underpin this process remain complex but include re-expression of a 'fetal gene program,' the induction of growth factors such as transforming growth factor- $\beta$, the re-expression of proto-oncogenes (such as c-fos) and the activation of neurohormones, including angiotensin-II, endothelin- 1 and norepinephrine. ${ }^{3}$ The resultant pathological changes seen include myocyte hypertrophy, local fibroblast proliferation and alterations in the myocardial extracellular matrix. ${ }^{4,5}$

It is widely accepted that blood pressure control is central in the prevention of the adverse effects of hypertension on the cardiovascular system. Furthermore, some recent studies have suggested that the use of antihypertensive therapies that modulate the renin-angiotensin system appears to afford a superior clinical benefit to that provided by blood pressure control alone. ${ }^{6,7}$ In this regard, the amount of renin-angiotensin system inhibition could offer potential benefit beyond blood pressure control. This protective effect of either angiotensin-converting enzyme inhibition or angiotensin receptor blockade is most likely mediated through the multiple described effects of angiotensin-II, ${ }^{8}$ but the exact molecular basis remains undefined.

Oligonucleotide microarray (gene chip) technology allows us to monitor large-scale changes in gene expression in biological systems. Here, we used Affymetrix oligonucleotide microarrays (Affymetrix, Santa Clara, CA, USA) to track changes in gene expression in the hearts of spontaneous hypertensive rats (SHRs) as a model of both early and established cardiac hypertrophy, with a view to further elucidate the molecular pathways involved in the pathogenesis of

${ }^{1}$ Cardiovascular Research Laboratory, Cardiology Department, Hospital General Universitario Gregorio Maranon, Madrid, Spain; ${ }^{2}$ Department of Medicine and Therapeutics, Conway Institute of Biomolecular and Biomedical Research, Mater Misericordiae University Hospital, University College Dublin and the Dublin Molecular Medicine Centre, Dublin, Ireland; ${ }^{3}$ Laboratory of Renal Physiopathology, Nephrology Department, Hospital General Universitario Gregorio Maranon, Madrid, Spain and ${ }^{4}$ Nephrology Department, Vascular Research Laboratory, Fundacion Jimenez Diaz, Autonoma University, Madrid, Spain

${ }^{5}$ Both junior and senior authors contributed equally to this work.

Correspondence: Professor J Egido, Vascular Research Laboratory, Fundacion Jimenez Diaz. Av/Reyes Catolicos 2; 28040 , Madrid, Spain.

E-mail: jegido@fjd.es

Received 13 November 2008; revised 26 December 2008; accepted 27 January 2009; published online 27 March 2009 
pressure-overload hypertrophy. Furthermore, we also examined the modulation of these changes in response to three different antihypertensive regimens. To maximize clinical relevance, the drug regimen was maintained for a wide period of the animal adulthood (11 months) to reflect the long-term effect of both hypertension and antihypertensive therapy on the heart.

\section{METHODS}

\section{Experimental model}

Studies were performed with male SHRs and normotensive Wistar-Kyoto (WKY) rats (Criffa, Barcelona, Spain). In order to increase renal damage SHRs underwent unilateral nephrectomy at 12 weeks of age. The animals were then randomized to the following groups, with six animals per group:

Untreated group: SHR followed without treatment (SHR48).

Quinapril group: SHR treated with $16 \mathrm{mg} \mathrm{kg}^{-1} \mathrm{day}^{-1}$ of the angiotensin-IIconverting enzyme inhibitor quinapril (QHI).

Combined quinapril plus the $\alpha$-adrenergic receptor blocker doxazosin treatment group: SHR receiving $15 \mathrm{mg} \mathrm{kg}^{-1} \mathrm{day}^{-1}$ doxazosin plus $1.6 \mathrm{mg} \mathrm{kg}^{-1} \mathrm{day}^{-1}$ of quinapril (DXQ).

Losartan group: SHR receiving $30 \mathrm{mg} \mathrm{kg}^{-1} \mathrm{day}^{-1}$ of the angiotensin-II type-1 receptor blocker losartan (LOS).

Doses were selected from an earlier pilot study showing the provision of adequate blood pressure control (systolic $\mathrm{BP}<140 \mathrm{~mm} \mathrm{Hg}$ ). Doxazosin and quinapril (as powdered hydrochloride salt) were kindly provided by Pfizer (Barcelona, Spain). Losartan was provided by Merck Sharp and Dohme (Madrid, Spain). All drugs were given in drinking water.

Animals were followed for 36 weeks after being included in the study at 12 weeks of age. However, to study 'early' changes in LVH, three animals from both control and SHR groups were sacrificed at the start of the study and designated the WKY12 and SHR12 groups, respectively. Animals were allowed to free water and food access in a controlled light, temperature and humidity environment.

Weekly systolic BP was measured by a tail-cuff sphygmomanometer (NARCO Biosystems, Austin, TX, USA). For each animal, the value of three independent systolic BP measurements was averaged and recorded at each session. The final average $\mathrm{BP}$ was the average of all averages recorded during the study.

At 48 weeks of age, the animals were sacrificed and the heart was removed and weighted. Cardiac index was calculated as the heart-to-body-weight ratio $\times 10^{3}$. Heart samples were obtained for both, fixed for $24 \mathrm{~h}$ in $4 \%$ paraformaldehyde and paraffin embedded and snap frozen in liquid nitrogen and then kept at $-80^{\circ} \mathrm{C}$ for further analysis.

The study was approved by the Institution Board for Experimental Animals and the guidelines of animal research were carefully followed.

\section{Statistical analysis}

The untreated SHR group was considered the control for the three treated SHR groups. To assess treatment-induced normalization of altered gene expression, the WKY group was considered as control (that is, healthy animals). Equality of variances of $\mathrm{BP}$ and hypertension-induced myocardial injury was tested with Levene's test. Means of normally distributed continuous variables with equal variances were analyzed with analysis of variance. If the variances were not equal, the Kruskal-Wallis test was used. A $P$-value $<0.05$ was considered significant. The tests were performed using the SPSS 11.5 software package (SPSS Inc., Chicago, IL, USA).

\section{Microarray experiments and bioinformatic analysis}

Tissue samples were obtained from the left ventricles of three animals per experimental group. The tissue was homogenized using the pestle and mortar technique and total RNA was extracted using Trizol (Invitrogen, Paisley, Scotland) and following the manufacturer's instructions. Five micrograms of total RNA was prepared for hybridization to the Affymetrix GeneChip Rat $230 \mathrm{~A}$ as described earlier. ${ }^{9}$ Each individual tissue sample was hybridized to its own chip, generating biological triplicates from each experimental group.

Image files were obtained through Affymetrix GeneChip software (Mas5). Subsequently, Robust Multichip Average (RMA) was performed. RMA is an
R-based technique that analyzes directly from the Affymetrix microarray *cel file and consists of three steps: background adjustment, quantile normalization and summarization. RMAexpress is a version compatible with a Windows operating system. As each experimental group was arrayed in triplicate, an average RMA value was computed. To ensure that the average was statistically representative, the standard error of the mean was calculated and average values with an s.e.m. exceeding 0.5 were excluded. A signal $\log$ ratio of $<-0.6$ and $>0.6$ was the cutoff used to signify differential expression. Using normalized RMA values, cluster analysis was performed using Eisen's program of Unsupervised Average Linkage Hierarchical Clustering, which essentially groups together genes that share a comparable expression pattern, through an algorithm based on a mathematical description of similarity (standard correlation coefficient) rather than through the magnitude of their absolute expression. Thus, genes with similar expression profiles are assembled into nodes, joined by branches, and the length of each branch is proportional to the degree of similarity between the expression profiles of the genes clustered within. ${ }^{10}$

\section{Principal component analysis}

Principal component analysis (PCA) was also performed using the Bioconductor and ADE4 packages from the open source statistical analysis software $\mathrm{R}$ (version 9.0, http://www.r-project.org/). PCA is an ordination technique that identifies the major trends in data sets. In essence, it seeks to represent the relationship of both row and column variables to result in a low-dimensional projection of an originally high-dimensional data set, and the distances among points in the projection set should resemble their original distances in the highdimensional space as closely as possible. ${ }^{11}$

\section{RESULTS}

\section{Blood pressure}

Twelve-week-old SHRs (SHR12) showed an average BP of $187 \pm 7 \mathrm{~mm} \mathrm{Hg}$, significantly higher $(P<0.001)$ than the same-aged WKY rats $(123 \pm 5 \mathrm{~mm} \mathrm{Hg})$. Non-treated SHRs displayed an average BP during the study of $219 \pm 7 \mathrm{~mm} \mathrm{Hg}$, significantly higher $(P<0.001)$ than all other treatment groups. The QHI, DXQ and LOS groups consistently had systolic BP readings less than $140 \mathrm{~mm} \mathrm{Hg}$, with an average BP of $121 \pm 8,125 \pm 8$ and $135 \pm 2 \mathrm{~mm} \mathrm{Hg}$ for QHI, DXQ and LOS, respectively. The Kruskal-Wallis test showed that all treatment groups had a significantly lower mean BP compared with the untreated SHR group. A post hoc analysis showed that the mean BP of the LOS group at the end of the study was significantly higher than that of the other two treatment groups $(P<0.05)$.

\section{Left ventricular hypertrophy}

The heart-to-body-weight ratio $\times 10^{3}$ (cardiac hypertrophy index) was analyzed as a surrogate marker of hypertension-induced LVH. Twelve-week-old SHRs showed a cardiac hypertrophy index of $3.9 \pm 0.1$, which was significantly higher than that of the age-matched controls $(3.0 \pm 0.1, P<0.001)$. By 48 weeks of age, the cardiac index of the SHR group had increased further in comparison with the 12-week-old SHRs $(P<0.01)$ and was also significantly higher than the age-matched normotensive controls WKY48 (4.6 \pm 0.8 vs. $2.8 \pm 0.4$ for SHR48 and WKY48, respectively, $P<0.001)$. All treatment groups showed an improvement in cardiac index values $(3.1 \pm 0.3,3.7 \pm 0.7$ and $3.6 \pm 0.5$ for QHI, DXQ and LOS, respectively) that was significantly lower than that in untreated animals.

\section{Heart injury score}

Cardiac pathological analysis showed extensive fibrotic and inflammatory foci in the SHR48 group $(4.3 \pm 1.4)$, and again each treatment regimen was associated with a significant reduction in this injury score $(P<0.01)$. In contrast, both WKY12 and SHR12 groups did not show significant heart injury ( $0.0 \pm 0.0$ vs. $1.0 \pm 1.0$, respectively), suggesting that, at an early stage of $\mathrm{LVH}$, pathological changes are not yet appreciated. 


\section{Global changes in gene expression in $\mathrm{LVH}$}

Average linkage hierarchical clustering of both individual microarray gene expression values ( $n=3$ for each condition) and also the RMAcomputed average resulted in the separation of the experimental groups, WKY12, SHR12, WKY48 and SHR48, into four distinct clusters as seen in Figure 1 (panel a). This would suggest that each group has its own distinct transcriptome and that the computed average RMA expression value is representative of each experimental group.

To identify hypertrophy-related changes in gene expression, comparison was made between the SHR12 and WKY12 groups and also between SHR48 and WKY48 to determine changes in both 'early' and 'late' or established $\mathrm{LVH}$, respectively. Of the 15696 gene sequences expressed on the Affymetrix GeneChip Rat-230A, there was a significant alteration (defined as signal $\log$ ratio $(\mathrm{SLR}) \leqslant-0.6$ or $\geqslant 0.6$ ) in the expression of 295 (1.9\%) of these transcripts in 'early' LVH, and of $143(0.9 \%)$ in 'late' or established LVH. Of the 295 transcripts altered in early LVH, 190 were upregulated, whereas 105 were downregulated (Table 1). Of the 143 genes altered in late LVH, 110 were upregulated and 33 were downregulated (Table 2).

Figure 1 (panel b) summarizes the changes seen in gene expression in the five most prominent functional classes (as defined by the OntoExpress Gene Ontology Database ${ }^{12}$ ) and includes genes involved in cellular metabolism/energetics, cell proliferation, signal transduction, development and muscle contraction/cytoskeleton function. Of these altered functional classes, the class of metabolic/energetic genes a
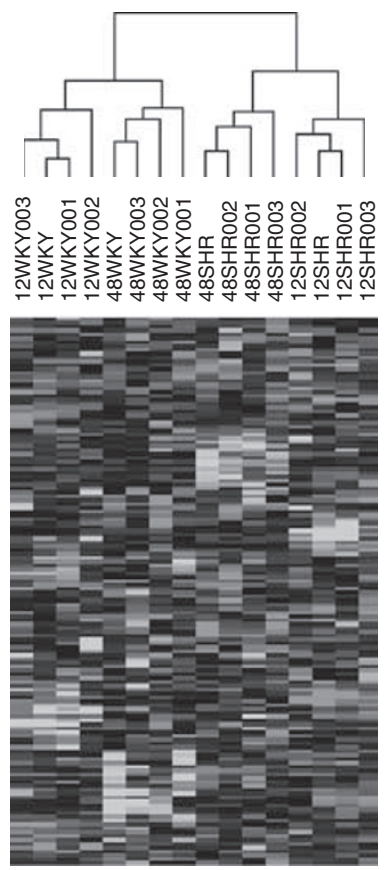

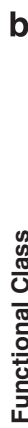

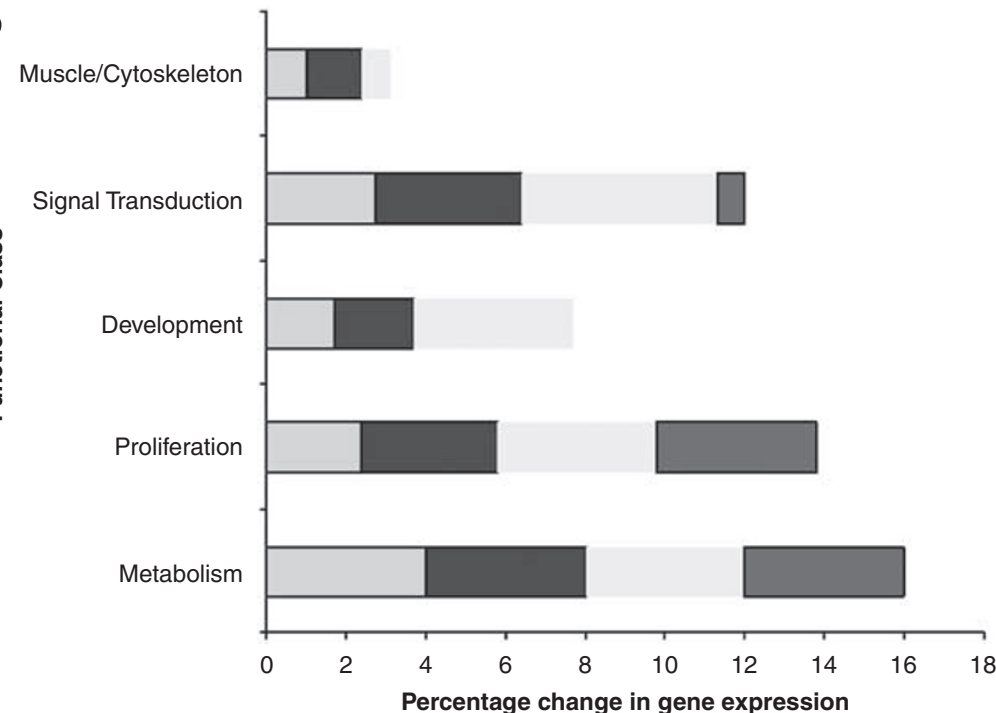

Figure 1 Global transcriptomic response. (a) Hierarchical cluster analysis of the individual and average arrays of both control groups, WKY12 and WKY48, and both LVH groups, SHR12 and SHR48, which separates the arrays into four distinct clusters corresponding to each group. (b) The changes in expression in the major gene function families as defined by Onto-Express. A full colour version of this figure can be found at the journal of Hypertension Research online. 
Table 1 Genes that show the most significant differential expression (as defined by the magnitude of signal log ratio) in early LVH

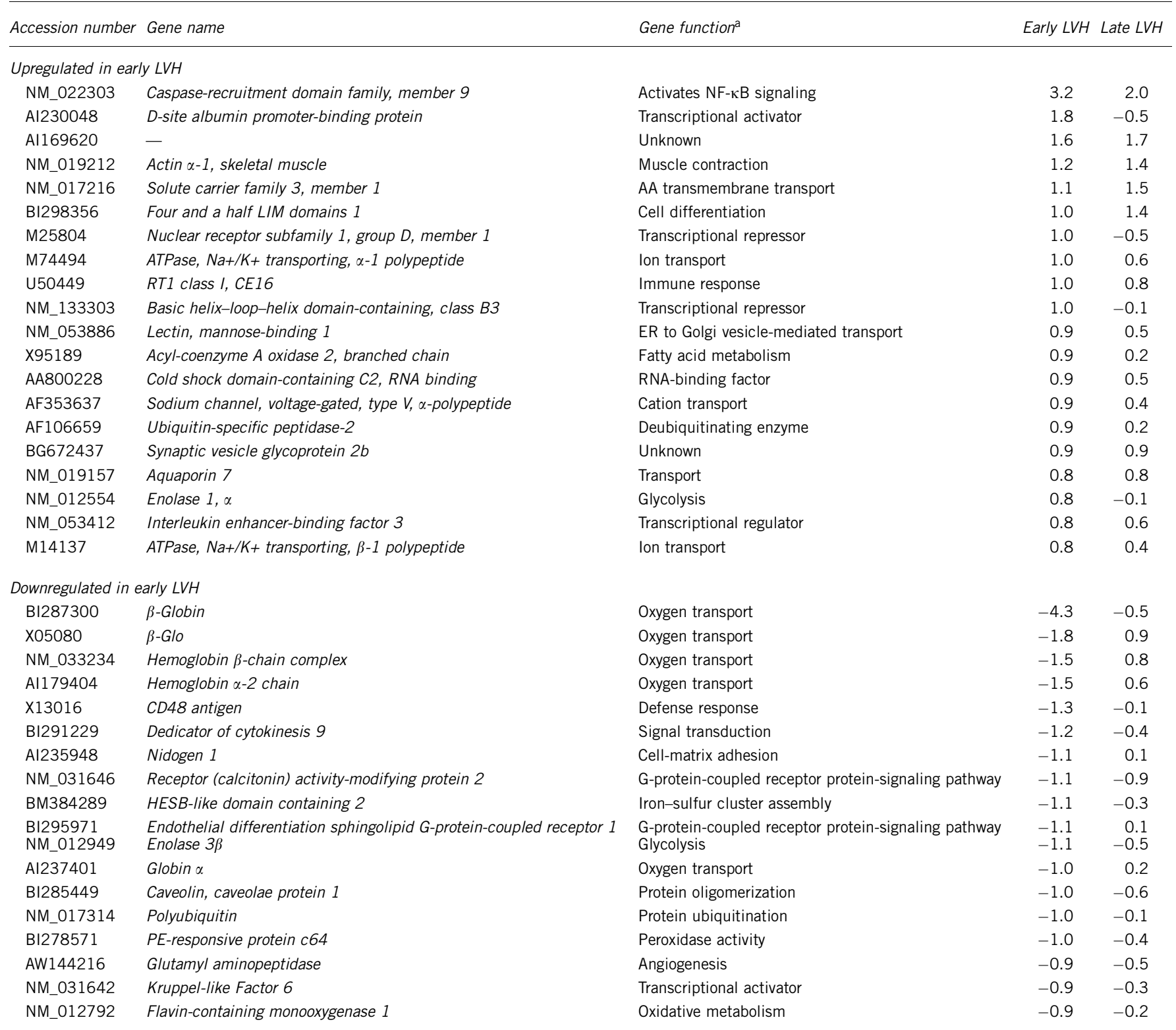

Abbreviations: LVH, left ventricular hypertrophy; SLR, signal log ratio.

aGene function derived from ONTO database (http://vortex.cs.wayne.edu/projects.htm) and literature review.

displayed the greatest perturbation at all stages of hypertrophy, followed by genes implicated in cellular proliferation and signal transduction, respectively. Smaller changes were seen in the development and muscle/cytoskeleton classes. Hierarchical cluster analysis ${ }^{10}$ was performed on these five functional cohorts, enabling visualization of the change in gene expression (whereby red signifies an increase in gene expression and green a decrease) in early and late LVH and also the subsequent modulation by the three different antihypertensive regimens (Figures 2 and 3).

\section{Modulation of the transcriptomic response by antihypertensive therapy}

To globally describe the relationship between the transcriptome of the control or 'normal' heart (WKY48) and that of the treated hypertensive heart, PCA was performed. Interestingly, despite the favorable hemodynamic profile of the three treatment groups, they still remained at a significant distance from control (Figure 4, panel a). The treatment group that showed the nearest distance (and hence most similarity in its molecular profile) to control was the QHI treatment group, the group with better BP control (Figure 5).

The number of altered transcripts normalized (normalization was empirically defined as showing the expression of the transcripts in a close range of the control (WKY48) value) by each treatment is shown in Figure 4 (panel b).

Only three transcripts, differentially expressed in late LVH, were normalized by all the three treatment regimens. The number of altered transcripts not modified by treatment is shown in Figure 4 (panel c). 
Table 2 Genes that show the most significant differential expression (as defined by the magnitude of signal log ratio) in late LVH

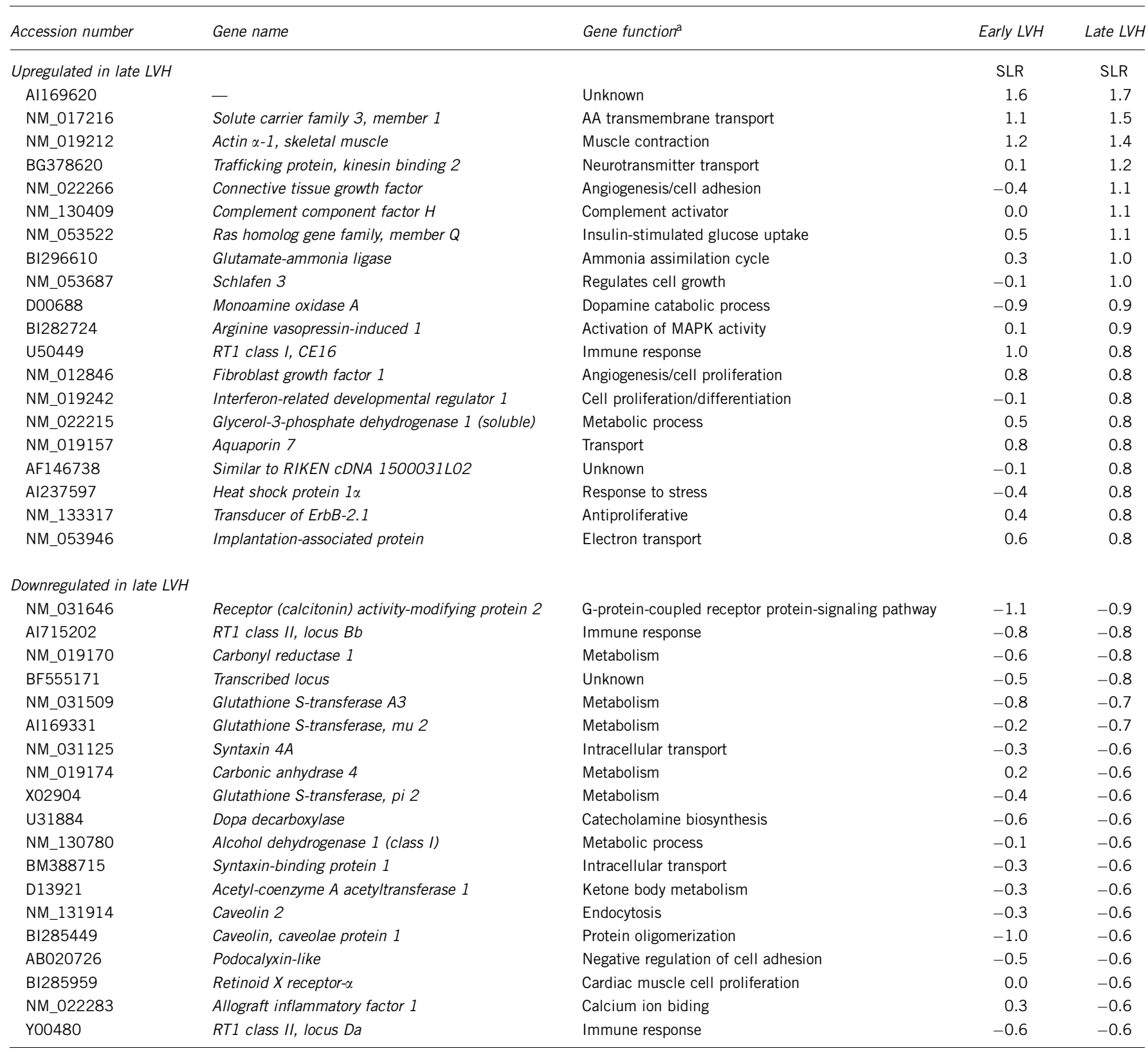

Abbreviations: LVH, left ventricular hypertrophy; SLR, signal log ratio.

aGene function derived from ONTO database (http://vortex.cs.wayne.edu/projects.htm) and literature review.

\section{DISCUSSION}

In this study, the transcriptomic response of the heart to sustained hypertension in vivo was identified, and analysis at two separate time points permitted the elucidation of temporal patterns in changes in gene expression between early and late/established LVH. Furthermore, the modulation of the molecular response of the hypertensive heart in vivo to three different effective antihypertensive treatment regimens was also identified.

\section{Metabolics/energetics}

The largest functional class showing differential expression in LVH was the metabolics/energetics class (Figure 2, panel a), a finding consistent with the known metabolic consequences of a sustained increase in cardiac workload. Under normal conditions, $60-90 \%$ of ATP forma- tion is derived from fatty acid oxidation (FAO) and $10-30 \%$ is derived from oxidation of pyruvate, which comes from glycolysis and lactate oxidation in approximately equal amounts. In earlier studies in $\mathrm{LVH}$, we and others have found a shift from FAO to increased rates of glucose oxidation, similar to that in the fetal heart, resulting in an improved cardiac efficiency ${ }^{13,14}$ as lower oxygen consumption cost per mole of ATP is generated, compared with FAO. This energy metabolic response may contribute in the long term to adverse consequences on cardiac function owing to diminished energy reserves and a reduced capacity to maintain myocyte lipid balance, as described in children with genetically determined deficiencies in FAO enzymes. ${ }^{15}$ In this study, there was a striking downregulation of a number of genes involved in the FAO pathway, mainly in early $\mathrm{LVH}$, in keeping with this earlier recognized metabolic switch. These included a decrease in 
a
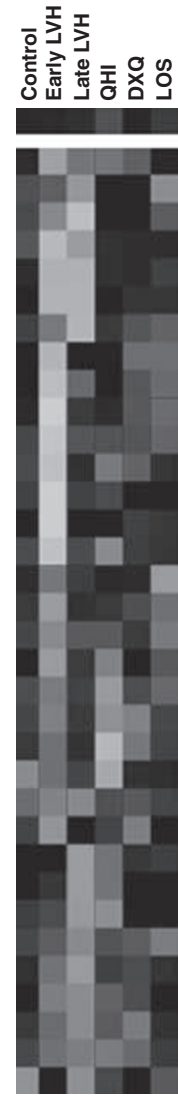

acetyl-Coenzyme A dehydrogenase, medium chain adenylate kinase

solute carrier family 3, member

pyruvate dehydrogenase kinase, isoenzyme 4

nudix-type motif 7 (predicted)

ankyrin repeat domain 23 (predicted)

glycerol-3-phosphate dehydrogenase 1 (soluble)

lipase, hormone sensitive

hexosaminidase $B$

3-hydroxybutyrate dehydrogenase, heart

dicarbonyl L-xylulose reductase

aminolevulinic acid synthase 1

ADP-ribosylation factor-like 6 interacting protein 5

muscle glycogen phosphorylase

citrate synthase

ADP-ribosylation factor 1

enolase 1, alpha

acyl-Coenzyme A oxidase 2, branched chain

glutamate oxaloacetate transaminase 1

fatty acid binding protein 4 , adipocyte

3-alpha-hydroxysteroid dehydrogenase

prominin 1

very low density lipoprotein receptor

endothelial differentiation sphingolipid GPCR1

aminolevulinic acid synthase 2

enolase 3, beta

acetyl-Coenzyme $A$ dehydrogenase

acetyl-coenzyme $A$ acetyltransferase 1

dopa decarboxylase

fatty acid binding protein 5, epiderma

glutathione S-transferase $A 3$

carbonyl reductase 1

glutathione S-transferase, pi 2

fatty acid binding protein 3
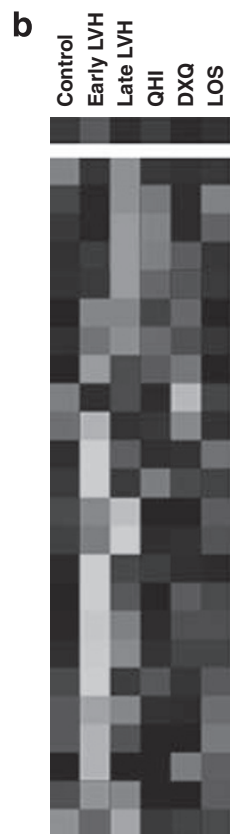

epithelial membrane protein

connective tissue growth factor

interferon-related development regulator 1

pre-B-cell colony enhancing factor 1

lysophospholipase 1

fibroblast growth factor

interleukin 6 signal transducer

thymoma viral proto-oncogene 2 (Akt2)

cyclin D2

insulin-like growth factor 2

serum/glucocorticoid regulated kinase

$C D C$-like kinase 1

carbonyl reductase 1

casein kinase II, alpha 1 polypeptide

flavin containing monooxygenase 1

cyclin I (predicted)

caveolin, caveolae protein 1

transketolase

interferon gamma receptor 1

eukaryotic translation elongation factor 1 alpha

glutamyl aminopeptidase

thioredoxin interacting protein

$D$ site albumin promoter binding protein

basic helix-loop-helix domain containing, class B3

Figure 2 Function-focused hierarchical cluster analysis. The transcripts that showed significant differential expression in early and/or late LVH were grouped into the functional cohorts of energy/metabolism (a) and cell growth/proliferation (b) and then their gene expression pattern, including modulation by the three antihypertensive treatments, is shown using hierarchical cluster analysis, in which green represents a decrease in gene expression and red an increase. A full colour version of this figure can be found at the journal of Hypertension Research online. a
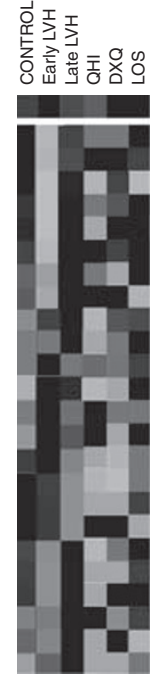

tyrosine 3-monooxygenase

Mitogen activated protein kinase 1

endothelial differentiation sphingolipid G-protein-coupled receptor 1

RAB28, member RAS oncogene family

ras homolog gene family, member $B$

kinase insert domain protein receptor

death-associated protein

secretin receptor

coagulation factor II (thrombin) receptor

calmodulin 1

receptor (calcitonin) activity modifying protein 2

thymoma viral proto-oncogene 1 (Akt1)

mitogen activated protein kinase kinase kinase 12

adrenergic receptor, alpha $1 d$

guanine nucleotide binding protein, alpha 12

thymoma viral proto-oncogene 2 (Akt2)

G-protein signalling modulator 1

endothelin receptor type $B$

ras homolog gene family, member $Q$

Rho-associated coiled-coil forming kinase 2

protein tyrosine phosphatase, receptor type,

proprotein convertase subtilisin/kexin type 6

heme oxygenase 1

RAS-related protein $1 \alpha$

b
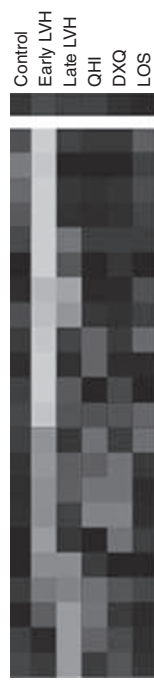

secretin recepto

guanine nucleotide binding protein (G protein), gamma 11

death-associated protein

Kruppel-like factor 6

paxillin

kinase insert domain protein receptor

olfactory receptor 1733

receptor (calcitonin) activity modifying protein 2

diacylglycerol kinase zeta

ras homolog gene family, member $B$

$E G F$, latrophilin and seven transmembrane domain containing 1

endothelial differentiation sphingolipid G-protein-coupled receptor 1

ankyrin 1, isoform 5

guanine nucleotide binding protein, alpha 12

G-protein signalling modulator 1

similar to Small EDRK-rich factor 2 (4F5rel)

adrenergic receptor, alpha $1 d$

Down syndrome critical region genel-like 1

transglutaminase 2, C polypeptide

ras homolog gene family, member $Q$

RAS-related protein $1 a$

calcium/calmodulin-dependent protein kinase I

C
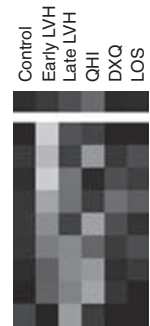

tropomyosin 1, alpha

vimentin

myosin $\mathrm{lb}$

tropomyosin 4

unconventional myosin Myr2 I heavy chain

actin alpha 1

enabled homolog (Drosophila)

enabled homo

restin

Figure 3 Function-focused hierarchical cluster analysis. The transcripts that showed a significant differential expression in early and/or late LVH were grouped into the functional cohorts of signal transduction (a), developmental processes (b) and muscle/cytoskeleton (c), and then their gene expression pattern, including modulation by the three antihypertensive treatments, is shown using hierarchical cluster analysis, in which green represents a decrease in gene expression and red an increase. A full colour version of this figure can be found at the journal of Hypertension Research online.

the fatty-acid-binding proteins 3, 4 and 5, which are essential for transport of fatty acids between the plasma membrane and mitochondria or peroxisomes for $\beta$-oxidation. ${ }^{16}$ In addition, there was also a decrease in the expression of the gene for the VLDL receptor, which is thought to provide cardiomyocytes to fatty acids access contained 
a

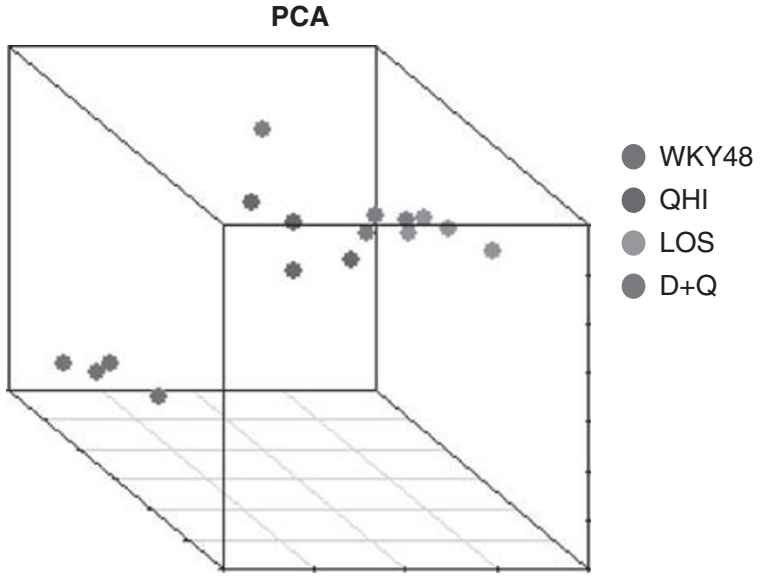

b

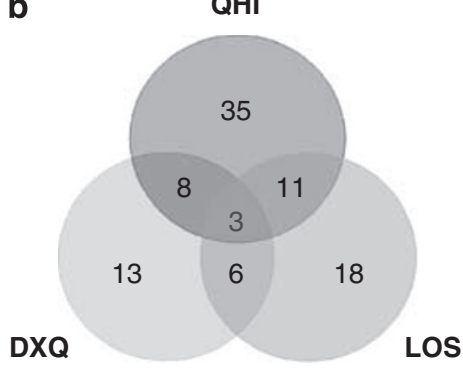

C

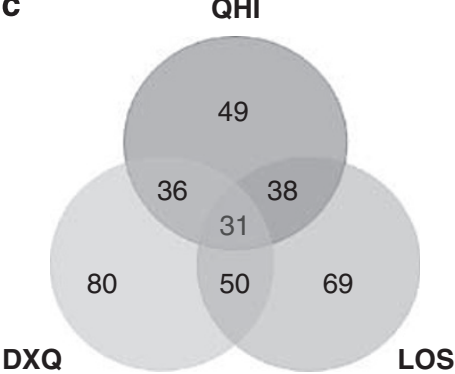

Figure 4 Pharmacogenomic response. (a) Principal component analysis was used to examine the relationship of the three treatment regimens (QHI, DXQ and LOS) with control (WKY48). Although there was a distinct separation of control from the three treatment groups, QHI was nearest in distance (and hence most similar) to control. (b) Normalization of LVH-associated transcripts by treatment. The Venn diagram illustrates the number of significantly differentially expressed transcripts in late LVH (total=143) that were normalized in the individual treatment groups. The overlapping segments indicate the number of transcripts in common between treatment groups. (c) Analysis of LVH-associated transcripts not altered by treatment. The Venn diagram illustrates the number of significantly differentially expressed transcripts in late LVH (total $=143$ ) that were not altered in response to the individual treatment regimens. The overlapping segments indicate the number of transcripts in common between treatment groups. A full colour version of this figure can be found at the journal of Hypertension Research online.

in circulating triglyceride-rich lipoproteins. ${ }^{17}$ Coincident with these changes, there was also downregulation of the acetylCoA dehydrogenase gene, an important catalyst of the first step in the $\beta$-oxidation process. In parallel, a switch to glucose oxidation in early LVH was suggested by the increase in expression of a key glycolytic enzyme, enolase 1- $\alpha$, and also of the glycogenolytic enzyme muscle glycogen phosphorylase. Interestingly, these changes were less evident in late/established $\mathrm{LVH}$, although there was a sustained decrease in the expression of fattyacid-binding protein 5 and also acetyl-coenzyme $A$ thiolase, which is important in peroxisome FAO. We also show an increased pyruvate dehydrogenase kinase 4 (PDK4) mRNA expression, the main inhibitor of cardiac pyruvate dehydrogenase, the key catalyst in pyruvate oxidation, ${ }^{18}$ seen in both early and established LVH. This finding is in contrast to other studies of cardiac hypertrophy in which PDK4 expression was found to be either decreased or unchanged, ${ }^{19,20}$ but it does correlate with earlier evidence of an imbalance in the coupling of glucose oxidation to glycolysis in hypertrophied hearts compared with non-hypertrophied hearts despite enhanced rates of pyruvate generation. ${ }^{21}$ It is possible that the increased PDK4 expression seen here may act as a brake on the flux of glucose through the pyruvate oxidation pathway, resulting in a mismatch in the rates of accelerated glycolysis and pyruvate oxidation in LVH. Interestingly, this mismatch, which may result in depleted energy reserves, has been postulated to contribute to contractile dysfunction seen in hypertrophied hearts, especially during reperfusion after ischemia. ${ }^{22}$ All three antihypertensive regimens tended to mitigate these changes.

\section{Proliferation/cell growth}

The cell proliferation/growth class was the second largest gene class showing a perturbation in expression in both early and established $\mathrm{LVH}$, which is unsurprising in view of the structural remodeling that occurs in LVH, including myocyte growth/hypertrophy and modulation of fibroblasts into a myofibroblast phenotype with concomitant proliferation. ${ }^{4}$ In this study, an increase was seen in akt2 and interleukin-6 transducer/gp130 expression in both early and established $\mathrm{LVH}$, which is consistent with an earlier study that has shown that the interleukin-6 family of cytokines contributes significantly to angiotensin-II-induced cardiac hypertrophy ${ }^{23}$ and that their receptor, Gp130, is critical for the development of pressure-overload $\mathrm{LVH}^{24}$ with the PI-3-kinase/akt pathway as an important downstream transducer of these effects. Akt itself is an important regulator of myocyte growth and survival, ${ }^{25}$ and constitutive overexpression of Akt in transgenic mice has been found to lead to increased contractility ${ }^{26}$ and pathological cardiac hypertrophy. ${ }^{27,28}$ Clustered with $A k t$ and gp130 was fibroblast growth factor-1, an angiogenic growth factor that has earlier been shown in human cardiac hypertrophy ${ }^{29}$ and that may reflect that blood vessel recruitment is an important feature of normal tissue growth. ${ }^{30} \mathrm{An}$ increase in connective tissue growth factor (CTGF) expression was seen in late $\mathrm{LVH}$, similar to findings in other models of cardiac hypertrophy, ${ }^{31}$ and its well-characterized profibrotic effect, including its ability to induce cardiac fibroblast proliferation and also extracellular matrix expansion. ${ }^{32}$ Hayata et al. ${ }^{33}$ have shown that cardiac myocytes stimulated with CTGF and its C-terminalregion peptide showed an increase in the cell surface area. Furthermore, they show that the inhibition of Akt abrogated CTGF-induced hypertrophy, indicating that CTGF is a novel hypertrophic factor in cardiac myocytes. ${ }^{33}$ In addition, antihypertensive treatment had a mitigating effect on the changes in gene expression in this group. As expected, CTGF expression, which is known to be angiotensin-II driven, was downregulated to a similar degree by both the QHI and LOS treatment groups, despite the blood pressure difference between these two groups. ${ }^{34-36}$ The combined DXQ treatment group did not show such effects, showing the angiotensin-II doseinhibition effect.

Interestingly, we have found a downregulation of the thioredoxininteracting protein (Txnip) in early LVH but not in late LVH. Overexpression of Txnip reduced protein synthesis in response to mechanical strain, phenylephrine or angiotensin-II. ${ }^{37}$ This protein is an endogenous thioredoxin inhibitor, which is a major intracellular antioxidant. However, reduced Txnip expression in the aortic con- 


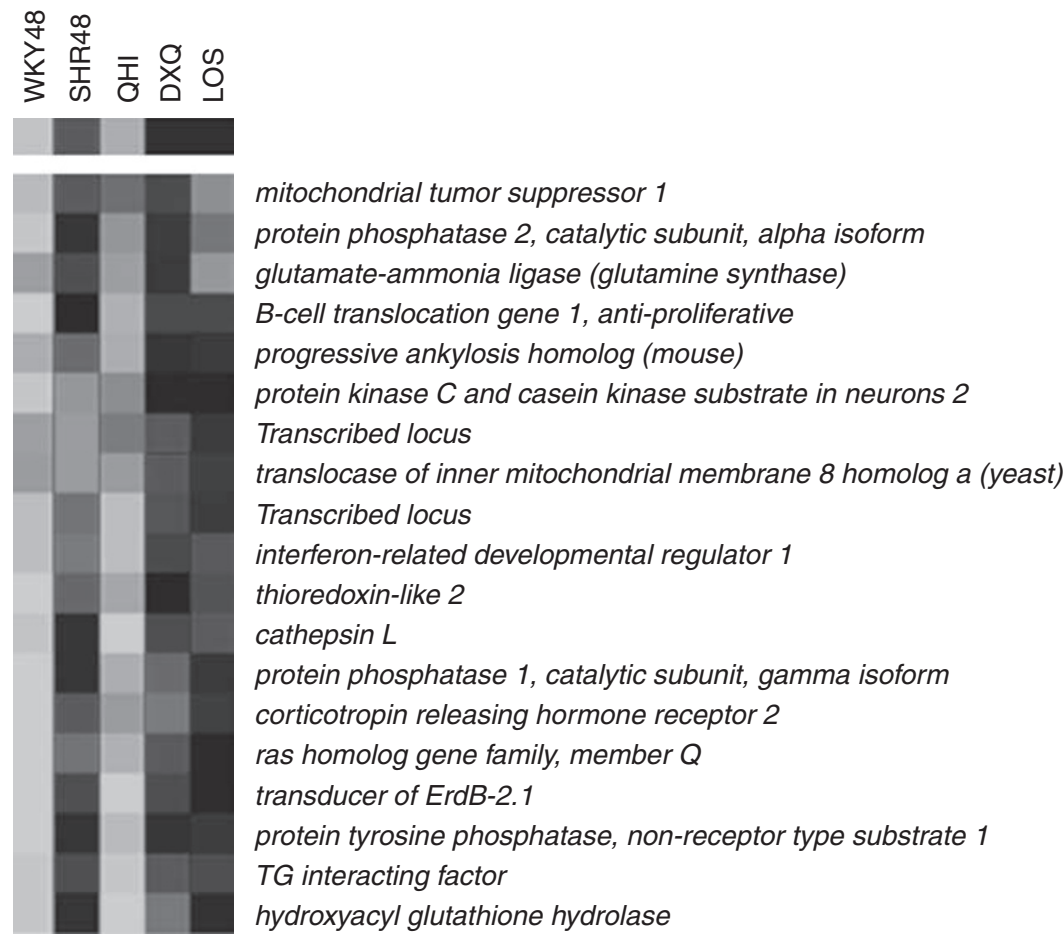

Figure 5 Cluster analysis of genes selectively normalized by QHI in late LVH. The transcripts differentially expressed in late LVH were then filtered to select out those selectively normalized by the QHI treatment group and average linkage hierarchical cluster analysis of this cohort was then performed to graphically illustrate their expression profile. A full colour version of this figure can be found at the journal of Hypertension Research online.

striction model increased the activation of thioredoxin, which participates in the development of cardiac hypertrophy. ${ }^{37}$ Furthermore, Txnip is required to maintain sufficient thioredoxin NADPH activity to reductively reactivate oxidized PTEN and oppose Akt downstream signaling. In fact, Txnip knockout mice showed increased Akt signaling and glycolysis in oxidative tissues (skeletal muscle and heart). ${ }^{38}$ Against this background, we have seen earlier that SHR48 presents an important oxidative stress compared with WKY48. ${ }^{39}$ Thus, Txnip could play a key role not only in cardiac hypertrophy but also in the transition from compensated to pathological LVH in a cross talk between Ang II/Akt/CTGF and Txnip/Akt/oxidative stress.

\section{Signal transduction}

Myocardial G-protein-coupled receptors, including the adrenergic, angiotensin and endothelin receptors, play an important role in hypertrophic growth by coupling with GTP-binding proteins, $\mathrm{G}_{\mathrm{s}}$, $\mathrm{G}_{\mathrm{q}} / \mathrm{G}_{11}$ and $\mathrm{G}_{\mathrm{i}}{ }^{40}$ The small $\mathrm{G}$ proteins, such as Ras, Rho and Rab, may also provide the critical link between these cell membrane receptors and the various signaling pathways regulating diverse cellular processes, such as cell growth, division and survival, organization of the cytoskeleton, membrane trafficking and cellular motility.

Signaling pathways evoked in LVH included the serine/threonine kinase Akt (1 and 2), the role of which in LVH has been well documented. ${ }^{26,33}$ Two G-protein-coupled receptors were also

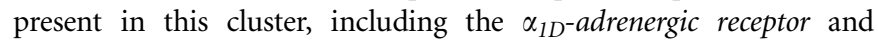
the G-protein-signaling modulator 1 (also known as AGS3). These aligned with the ubiquitously expressed guanine nucleotide-binding protein, $\alpha-12\left(\mathrm{G}_{\alpha} 12\right)$, which can couple with a wide range of receptors, including angiotensin-II and endothelin. ${ }^{41}$ The activation of $\mathrm{G}_{\alpha} 12$ can result in a variety of cellular responses, including cellular proliferation, neoplastic transformation and cytoskeletal reorganization. Furthermore, it has been implicated in mediating $\alpha_{1}$-adrenergic receptor-induced cardiac hypertrophy. ${ }^{42}$ Moreover, it has been found that the overexpression of AGS3 alters the surface ratios of heterologously expressed plasma membrane receptors and channels, and affects the internalization or recycling of surface proteins. ${ }^{43}$

As mentioned earlier, the small G proteins, such as Ras, Rho and Rab, are the likely downstream targets after activation of these receptors, and there is considerable interest in these proteins both as mediators of myocyte hypertrophy and as therapeutic targets. ${ }^{44-46}$ Against this background, it is interesting that a significant proportion of the cluster of signal transduction-related genes showing increased expression in late LVH belong to the small GTPase family and included the ras homolog gene family, member $Q$ (also known as TC10 or Rhoq), Rho-associated coiled-coil forming kinase 2 (ROCK2) and $R A S$-related protein 1 a (Figure $3 \mathrm{a}$ ). It has been shown that the $\mathrm{Cbl} /$ CAP/TC10 insulin signaling pathway is active in cardiac muscle and is impaired during obesity and insulin deficiency, ${ }^{47}$ but to our knowledge, this is the first time that TC10 is related to both hypertension and heart hypertrophy. Furthermore, Rho B, which, unlike other members of the Rho family, inhibits cell growth and proliferation and is negatively regulated by oncogenes including Ras, showed decreased expression in early LVH, adding further support for the importance of this G-protein signaling pathway in LVH. In the same cluster, we have seen a downregulation in the expression of the mitogen-activated protein kinase 1 (also called extracellular signalrelated kinase-2). There is a large body of evidence showing an activation of extracellular signal-related kinase- $1 / 2$ in response to many known hypertropic agonists. ${ }^{48,49}$ However, Purcell et al. ${ }^{50}$ have recently shown that extracellular signal-related kinase-1/2 signaling is not required for mediating physiological or pathological cardiac hypertrophy in vivo; moreover, blockade or deletion of cardiac 
Table 3 LVH-associated transcripts, the expression of which is not modified by any treatment group

\begin{tabular}{|c|c|c|}
\hline Accession no. & Upregulated transcripts in $\mathrm{LVH}$ & $S L R$ \\
\hline BM387112 & Transcribed locus & 0.9 \\
\hline Al549393 & Transcribed locus & 0.9 \\
\hline Al639523 & Spectrin $\alpha 1$ & 0.9 \\
\hline Al407930 & Protein kinase $C$ and casein kinase substrate in neurons 2 & 0.8 \\
\hline BI294716 & Transcribed locus & 0.8 \\
\hline BF285466 & Hypothetical protein LOC680259 & 0.8 \\
\hline U50449 & RT1 class I, CE16 & 0.8 \\
\hline M62763 & sterol carrier protein 2 & 0.7 \\
\hline BG666709 & $\mathrm{N}$-myc downstream-regulated gene 4 & 0.7 \\
\hline Al232036 & ATPase, $\mathrm{Na}+/ \mathrm{K}+$ transporting $\beta-1$ polypeptide & 0.7 \\
\hline NM_017079 & CD1d1 antigen & 0.7 \\
\hline AB007688 & Homer homolog 1 (Drosophila) & 0.7 \\
\hline NM_016986 & Acetyl-coenzyme A dehydrogenase, medium chain & 0.7 \\
\hline NM_012699 & DnaJ (Hsp40) homolog, subfamily B, member 9 & 0.6 \\
\hline Al138048 & Down syndrome critical region gene 1-like 1 & 0.6 \\
\hline AF367467 & Ratsg2 & 0.6 \\
\hline Al170664 & PAP-associated domain-containing 5 (predicted) & 0.6 \\
\hline Al230548 & Transcribed locus & -1.4 \\
\hline BI295153 & Transcribed locus, strongly similar to XP_214980.3 & -1.4 \\
\hline NM_031646 & Receptor (calcitonin) activity-modifying protein 2 & -0.9 \\
\hline Al715202 & RT1 class $I I$, locus $B b$ & -0.8 \\
\hline NM_019170 & Carbonyl reductase 1 & -0.8 \\
\hline BF555171 & Transcribed locus & -0.8 \\
\hline NM_031509 & Glutathione S-transferase A3 & -0.7 \\
\hline Al411100 & Nitrilase family, member 2 & -0.7 \\
\hline BI297693 & Transcribed locus & -0.7 \\
\hline $\mathrm{BI} 288816$ & Ras-related GTP-binding D (predicted) & -0.7 \\
\hline Al709531 & $\begin{array}{l}\text { Similar to cytochrome c oxidase polypeptide VIla-heart, } \\
\text { mitochondrial precursor }\end{array}$ & -0.6 \\
\hline NM_019174 & Carbonic anhydrase 4 & -0.6 \\
\hline U31884 & Dopa decarboxylase & -0.6 \\
\hline NM_130780 & Alcohol dehydrogenase 1 (class I) & -0.6 \\
\hline
\end{tabular}

Abbreviations: LVH, left ventricular hypertrophy; SLR, signal log ratio.

extracellular signal-related kinase-1/2 did predispose the heart to decompensation and failure after long-term pressure overload.

\section{Developmental genes in LVH}

It has earlier been appreciated that the cardiac response to increased work can result in the re-emergence of the fetal gene program. ${ }^{51}$ Several developmental genes were upregulated in both stages of hypertrophy including skeletal $\alpha$-actin 1, a well-recognized marker of cardiac hypertrophy, and the aforementioned DSCR1 and FGF1. Interestingly, there was also upregulation of two different regulators of muscle development, namely, four-and-a-half LIM domain 1 (FHL1), a LIM domain protein thought to play a role in skeletal muscle differentiation and identified earlier in genetic human hypertrophic cardiomyopathy, ${ }^{52}$ and also a regulator of myogenesis interferon-related developmental regulator. ${ }^{53}$

\section{Changes in muscle contraction/cytoskeleton}

Reorganization of the actin cytoskeleton and an increase in contractile proteins constitute well-recognized responses of the heart to hypertrophy. In this study, there was a relatively small change seen in the gene expression of the muscle contraction/cytoskeleton class, but it did include the upregulation of skeletal muscle $\alpha$-actin 1, unconven- tional myosin Myr2 I heavy chain (myolC) and also tropomodulin. Interestingly, tropomodulin 1 overexpression has earlier been implicated in the pathogenesis of a dilated cardiomyopathy. ${ }^{54}$ These changes were reversed by all three treatment regimens and have earlier been described by us using a proteomic analysis of $\mathrm{LVH}^{14}$

\section{Pharmacogenomics of $\mathrm{LVH}$ regression}

Principal component analysis showed that despite the favorable hemodynamic profile of the three treatment groups, they still remained at a significant distance from the control or normal heart (WKY48), suggesting that even with optimal BP treatment and associated LVH regression, the LVH transcriptome is not reversed, a finding in accordance with the histological evidence of inflammatory and fibrotic foci in all three treatment groups. Such a finding may suggest that regressed LVH may have clinically adverse consequences that may not be as somber as unchanged LVH, but that may probably compare unfavorably with hearts not going through an earlier hypertrophic phase, an issue not studied in clinical trials.

It is notable that 31 transcripts were unchanged by any treatment group and they may represent suitable targets for new research (Table 3). This cohort included genes involved in the actin cytoskeleton (for example, spectrin $1 \alpha$ ), signal transduction (for example, DSCR1, receptor activity-modifying protein 2), stress response (for example, dnaJ homolog, subfamily b, member 9) and metabolism (sterol carrier protein 2, acetyl-coenzyme A dehydrogenase, medium chain).

Figure 5 shows the LVH-associated genes that were normalized selectively by $\mathrm{QHI}$ in comparison with the other treatment groups. It is worth noting that this cohort included thioredoxin-like 2, which is intriguing, as a recent study has suggested that the antioxidant thioredoxin may paradoxically act as a significant growth promoter in the setting of pressure overload, possibly through a redox-dependent regulation of nuclear factor- $\kappa \mathrm{B}$ transcription. ${ }^{37}$ Another member of this cohort was the small G-protein ras homolog gene family, member $Q$ (or Tc10), which has also been implicated in muscle differentiation $^{55}$ similar to another member of this cohort, IFDR1. The relative contribution of these findings deserves further research.

Despite that all treatment groups showed a mean systolic BP below $140 \mathrm{~mm} \mathrm{Hg}$, the losartan-treated group had a higher mean BP in the post hoc analysis, which impedes direct comparison of this group with other treatment groups. Although this is a limitation of the study, the main comparison between the treated group and controls is still valid. Also, we are able to show the dose-specific effects of angiotensinconverting enzyme inhibition in the QHI $v$ s. the DXQ group at the molecular level and with the same mean BP.

In conclusion, molecular profiling of the hypertensive heart in vivo revealed a distinct LVH transcriptome, including prominent changes in gene expression in the following functional groups: metabolic, signal transduction, cell growth/proliferation, developmental and muscle contraction/cytoskeletal function. Antihypertensive treatment with a sustained reduction of BP in all three treatment groups was associated with regression of LVH and reduction in cardiac injury and also attenuation, for the most part, but not all of these gene expression changes. Nonetheless, even the transcriptome of the optimally treated hypertensive heart still differed substantially from that of the normotensive control on both a histological and molecular basis. These findings, supported by those obtained by a proteomic approach of $\mathrm{LVH}$ in this model, ${ }^{14}$ may deserve future clinical investigation.

\section{CONFLICT OF INTEREST}

The authors declare no conflict of interest. 


\section{ACKNOWLEDGEMENTS}

We appreciate the contribution of Pfizer Inc. for kindly providing quinapril and doxazosin. This work was supported by grants from the Health Research Board (to HR Brady, SB Connolly and NE Kieran), the European Union (HR Brady, P Doran and J Egido), the Irish Programme for Research in Third Level Institutions, the Spanish Cardiovascular network (RECAVA, RD06/0014/0035) and Comunidad Autonoma de Madrid (CAM, S-GEN-0247/2006), SAF (2007/ 63648) and P-BIO-0194-2006 CARDIOVREP-CM to Julio Osende. JGD is a recipient of a research contract from 'Juan de la Cierva' program from the Spanish Ministry of Science and Innovation.

1 Levy D. Left ventricular hypertrophy. Epidemiological insights from the Framingham Heart Study. Drugs 1988; 35(Suppl 5): 1-5.

2 Mosterd A, Cost B, Hoes AW, de Bruijne MC, Deckers JW, Hofman A, Grobbee DE. The prognosis of heart failure in the general population: The Rotterdam Study. Eur Heart $J$ 2001; 22: 1318-1327.

3 Depre C, Shipley GL, Chen W, Han Q, Doenst T, Moore ML, Stepkowski S, Davies PJ, Taegtmeyer $\mathrm{H}$. Unloaded heart in vivo replicates fetal gene expression of cardiac hypertrophy. Nat Med 1998; 4: 1269-1275.

4 Lips DJ, deWindt LJ, van Kraaij DJ, Doevendans PA. Molecular determinants of myocardial hypertrophy and failure: alternative pathways for beneficial and maladaptive hypertrophy. Eur Heart J 2003; 24: 883-896.

5 Lin J, Davis HB, Dai Q, Chou YM, Craig T, Hinojosa-Laborde C, Lindsey ML. Effects of early and late chronic pressure overload on extracellular matrix remodeling. Hypertens Res 2008; 31: 1225-1231.

6 Okin PM, Devereux RB, Jern S, Kjeldsen SE, Julius S, Nieminen MS, Snapinn S, Harris KE, Aurup P, Edelman JM, Wedel H, Lindholm LH, Dahlöf B;, LIFE Study Investigators. Regression of electrocardiographic left ventricular hypertrophy during antihypertensive treatment and the prediction of major cardiovascular events. JAMA 2004; 292: 2343-2349.

7 Wing LM, Reid CM, Ryan P, Beilin LJ, Brown MA, Jennings GL, Johnston Cl, McNeil JJ, Macdonald GJ, Marley JE, Morgan TO, West MJ;, Second Australian National Blood Pressure Study Group. A comparison of outcomes with angiotensin-converting-enzyme inhibitors and diuretics for hypertension in the elderly. N Engl J Med 2003; 348: 583-592.

8 Ruiz-Ortega M, Lorenzo O, Ruperez M, Esteban V, Suzuki Y, Mezzano S, Plaza JJ, Egido $\mathrm{J}$. Role of the renin-angiotensin system in vascular diseases: expanding the field. Hypertension 2001; 38: 1382-1387

9 Kieran NE, Doran PP, Connolly SB, Greenan MC, Higgins DF, Leonard M, Godson C, Taylor CT, Henger A, Kretzler M, Burne MJ, Rabb H, Brady HR. Modification of the transcriptomic response to renal ischemia/reperfusion injury by lipoxin analog. Kidney Int 2003; 64: 480-492.

10 Eisen MB, Spellman PT, Brown PO, Botstein D. Cluster analysis and display of genome-wide expression patterns. Proc Natl Acad Sci USA 1998; 95: 14863-14868.

11 Fellenberg K, Hauser NC, Brors B, Neutzner A, Hoheisel JD, Vingron M. Correspondence analysis applied to microarray data. Proc Natl Acad Sci USA 2001; 98 : 10781-10786.

12 Draghici S, Khatri P, Bhavsar P, Shah A, Krawetz SA, Tainsky MA. Onto-Tools, the toolkit of the modern biologist: Onto-Express, Onto-Compare, Onto-Design and Onto-Translate. Nucleic Acids Res 2003; 31: 3775-3781.

13 Huss JM, Kelly DP. Mitochondrial energy metabolism in heart failure: a question of balance. J Clin Invest 2005; 115: 547-555.

14 Gallego-Delgado J, Lazaro A, Osende JI, Barderas MG, Duran MC, Vivanco F, Egido J. Comparison of the protein profile of established and regressed hypertension-induced left ventricular hypertrophy. J Proteome Res 2006; 5: 404-413.

15 Lehman JJ, Kelly DP. Gene regulatory mechanisms governing energy metabolism during cardiac hypertrophic growth. Heart Fail Rev 2002; 7: 175-185.

16 van der Lee KA, Vork MM, De Vries JE, Willemsen PH, Glatz JF, Reneman RS, Van der Vusse GJ, Van Bilsen M. Long-chain fatty acid-induced changes in gene expression in neonatal cardiac myocytes. J Lipid Res 2000; 41: 41-47.

17 Masuzaki H, Jingami H, Matsuoka N, Nakagawa O, Ogawa Y, Mizuno M, Yoshimasa Y, Yamamoto T, Nakao K. Regulation of very-low-density lipoprotein receptor in hypertrophic rat heart. Circ Res 1996; 78: 8-14.

18 Stanley WC, Morgan EE, Huang H, McElfresh TA, Sterk JP, Okere IC, Chandler MP, Cheng J, Dyck JR, Lopaschuk GD. Malonyl-CoA decarboxylase inhibition suppresses fatty acid oxidation and reduces lactate production during demand-induced ischemia. Am J Physiol Heart Circ Physiol 2005; 289: H2304-H2309.

19 Lydell CP, Chan A, Wambolt RB, Sambandam N, Parsons H, Bondy GP, Rodrigues B, Popov KM, Harris RA, Brownsey RW, Allard MF. Pyruvate dehydrogenase and the regulation of glucose oxidation in hypertrophied rat hearts. Cardiovasc Res 2002; 53: $841-851$.

20 Taegtmeyer H, Razeghi P, Young ME. Mitochondrial proteins in hypertrophy and atrophy: a transcript analysis in rat heart. Clin Exp Pharmacol Physiol 2002; 29: 346-350.

21 Leong HS, Brownsey RW, Kulpa JE, Allard MF. Glycolysis and pyruvate oxidation in cardiac hypertrophy - why so unbalanced? Comp Biochem Physiol A Mol Integr Physiol 2003; 135: 499-513.
22 Sambandam N, Lopaschuk GD, Brownsey RW, Allard MF. Energy metabolism in the hypertrophied heart. Heart Fail Rev 2002; 7: 161-173.

23 Sano M, Fukuda K, Kodama H, Pan J, Saito M, Matsuzaki J, Takahashi T, Makino S, Kato T, Ogawa S. Interleukin-6 family of cytokines mediate angiotensin II-induced cardiac hypertrophy in rodent cardiomyocytes. J Biol Chem 2000; 275: 29717-29723.

24 Uozumi H, Hiroi Y, Zou Y, Takimoto E, Toko H, Niu P, Shimoyama M, Yazaki Y, Nagai R, Komuro I. gp130 plays a critical role in pressure overload-induced cardiac hypertrophy. J Biol Chem 2001; 276: 23115-23119.

25 Fujio Y, Nguyen T, Wencker D, Kitsis RN, Walsh K. Akt promotes survival of cardiomyocytes in vitro and protects against ischemia-reperfusion injury in mouse heart. Circulation 2000; 101: 660-667.

26 Condorelli G, Drusco A, Stassi G, Bellacosa A, Roncarati R, laccarino G, Russo MA, Gu Y, Dalton N, Chung C, Latronico MV, Napoli C, Sadoshima J, Croce CM, Ross Jr J. Akt induces enhanced myocardial contractility and cell size in vivo in transgenic mice. Proc Natl Acad Sci USA 2002; 99: 12333-12338.

27 Shioi T, McMullen JR, Kang PM, Douglas PS, Obata T, Franke TF, Cantley LC, Izumo S. Akt/protein kinase B promotes organ growth in transgenic mice. Mol Cell Biol 2002; 22: 2799-2809.

28 Matsui T, Li L, Wu JC, Nagoshi T, Picard MH, Liao R, Rosenzweig A. Phenotypic spectrum caused by transgenic overexpression of activated Akt in the heart. J Biol Chem 2002; 277: 22896-22901.

29 Tomita Y, Kusama Y, Seino Y, Munakata K, Kishida H, Hayakawa H. Increased accumulation of acidic fibroblast growth factor in left ventricular myocytes of patients with idiopathic cardiomyopathy. Am Heart J 1997; 134: 779-786.

30 Fernandez B, Buehler A, Wolfram S, Kostin S, Espanion G, Franz WM, Niemann H, Doevendans PA, Schaper W, Zimmermann R. Transgenic myocardial overexpression of fibroblast growth factor- 1 increases coronary artery density and branching. Circ Res 2000; 87: 207-213.

31 Chen H, Huang XN, Stewart AF, Sepulveda JL. Gene expression changes associated with fibronectin-induced cardiac myocyte hypertrophy. Physiol Genomics 2004; 18 : 273-283.

32 Leask A, Abraham DJ. TGF-beta signaling and the fibrotic response. FASEB J 2004; 18: 816-827.

33 Hayata N, Fujio Y, Yamamoto Y, Iwakura T, Obana M, Takai M, Mohri T, Nonen S, Maeda M, Azuma J. Connective tissue growth factor induces cardiac hypertrophy through Akt signaling. Biochem Biophys Res Commun 2008; 370: 274-278.

34 Rosenkranz S. TGF-beta1 and angiotensin networking in cardiac remodeling. Cardiovasc Res 2004; 63: 423-432.

35 Che ZQ, Gao PJ, Shen WL, Fan CL, Liu JJ, Zhu DL. Angiotensin II-stimulated collagen synthesis in aortic adventitial fibroblasts is mediated by connective tissue growth factor. Hypertens Res 2008; 31: 1233-1240.

36 Tsutsui H, Matsushima S, Kinugawa S, Ide T, Inoue N, Ohta Y, Yokota T, Hamaguchi S, Sunagawa K. Angiotensin II type 1 receptor blocker attenuates myocardial remodeling and preserves diastolic function in diabetic heart. Hypertens Res 2007; 30: 439-449.

37 Yoshioka J, Schulze PC, Cupesi M, Sylvan JD, MacGillivray C, Gannon J, Huang H, Lee RT. Thioredoxin-interacting protein controls cardiac hypertrophy through regulation of thioredoxin activity. Circulation 2004; 109: 2581-2586.

38 Hui ST, Andres AM, Miller AK, Spann NJ, Potter DW, Post NM, Chen AZ, Sachithanantham S, Jung DY, Kim JK, Davis RA. Txnip balances metabolic and growth signaling via PTEN disulfide reduction. Proc Natl Acad Sci USA 2008; 105: 3921-3926.

39 Lazaro A, Gallego-Delgado J, Justo P, Esteban V, Osende J, Mezzano S, Ortiz A, Egido J. Long-term blood pressure control prevents oxidative renal injury. Antioxid Redox Signal 2005; 7: 1285-1293.

40 Frey N, Olson EN. Cardiac hypertrophy: the good, the bad, and the ugly. Annu Rev Physiol 2003; 65: 45-79.

41 Neves SR, Ram PT, lyengar R. G protein pathways. Science 2002; 296: 1636-1639.

42 Yun J, Zuscik MJ, Gonzalez-Cabrera P, McCune DF, Ross SA, Gaivin R, Piascik MT, Perez DM. Gene expression profiling of alpha(1b)-adrenergic receptor-induced cardiac hypertrophy by oligonucleotide arrays. Cardiovasc Res 2003; 57: 443-455.

43 Groves B, Gong Q, Xu Z, Huntsman C, Nguyen C, Li D, Ma D. A specific role of AGS3 in the surface expression of plasma membrane proteins. Proc Natl Acad Sci USA 2007; 104: 18103-18108.

44 Ritchie RH, Marsh JD, Lancaster WD, Diglio CA, Schiebinger RJ. Bradykinin blocks angiotensin II-induced hypertrophy in the presence of endothelial cells. Hypertension 1998; 31: 39-44.

45 Satoh S, Ueda Y, Koyanagi M, Kadokami T, Sugano M, Yoshikawa Y, Makino N. Chronic inhibition of Rho kinase blunts the process of left ventricular hypertrophy leading to cardiac contractile dysfunction in hypertension-induced heart failure. J Mol Cell Cardiol 2003; 35: 59-70.

46 Barry SP, Davidson SM, Townsend PA. Molecular regulation of cardiac hypertrophy. Int J Biochem Cell Biol 2008; 40: 2023-2039.

47 Gupte A, Mora S. Activation of the Cbl insulin signaling pathway in cardiac muscle; dysregulation in obesity and diabetes. Biochem Biophys Res Commun 2006; 342: 751-757.

48 Bueno OF, Molkentin JD. Involvement of extracellular signal-regulated kinases $1 / 2$ in cardiac hypertrophy and cell death. Circ Res 2002; 91: 776-781.

49 Nakano S, Kobayashi N, Yoshida K, Ohno T, Matsuoka H. Cardioprotective mechanisms of spironolactone associated with the angiotensin-converting enzyme/epidermal growth factor receptor/extracellular signal-regulated kinases, $N A D(P) H$ oxidase/lectin-like oxidized low-density lipoprotein receptor-1, and Rho-kinase pathways in aldosterone/ salt-induced hypertensive rats. Hypertens Res 2005; 28: 925-936. 
50 Purcell NH, Wilkins BJ, York A, Saba-El-Leil MK, Meloche S, Robbins J, Molkentin JD. Genetic inhibition of cardiac ERK1/2 promotes stress-induced apoptosis and heart failure but has no effect on hypertrophy in vivo. Proc Natl Acad Sci USA 2007; 104: 14074-14079.

51 Chien KR, Knowlton KU, Zhu H, Chien S. Regulation of cardiac gene expression during myocardial growth and hypertrophy: molecular studies of an adaptive physiologic response. FASEB J 1991; 5: 3037-3046.

52 Lim DS, Roberts R, Marian AJ. Expression profiling of cardiac genes in human hypertrophic cardiomyopathy: insight into the pathogenesis of phenotypes. J Am Coll Cardiol 2001; 38: 1175-1180.
53 Micheli L, Leonardi L, Conti F, Buanne P, Canu N, Caruso M, Tirone F. PC4 coactivates MyoD by relieving the histone deacetylase 4-mediated inhibition of myocyte enhancer factor 2C. Mol Cell Biol 2005; 25: 2242-2259.

54 Sussman MA, Welch S, Gude N, Khoury PR, Daniels SR, Kirkpatrick D, Walsh RA, Price RL, Lim HW, Molkentin JD. Pathogenesis of dilated cardiomyopathy: molecular, structural, and population analyses in tropomodulin-overexpressing transgenic mice. Am J Pathol 1999; 155: 2101-2113.

55 Abe T, Kato M, Miki H, Takenawa T, Endo T. Small GTPase Tc10 and its homologue RhoT induce N-WASP-mediated long process formation and neurite outgrowth. J Cell Sci 2003; 116: 155-168. 